\title{
The Role of TLR4 in M1 Macrophage- Induced Epithelial-Mesenchymal Transition of Peritoneal Mesothelial Cells
}

\author{
Jun Shi Qing Li Meixiao Sheng Min Zheng Manshu Yu Lu Zhang \\ First Affiliated Hospital of Nanjing University of Chinese Medicine, Nanjing, China
}

\section{Key Words}

Macrophage $\cdot$ Peritoneal mesothelial cells $•$ EMT $•$ TLR4

\begin{abstract}
Background/Aims: Peritoneal fibrosis is a frequent complication of peritoneal dialysis that follows inflammation. It is recognized that epithelial-mesenchymal transition (EMT) of peritoneal mesothelial cells (PMCs), plays a key role in fibrogenesis. However, the relationship between inflammatory macrophages and PMCs remains elusive. In this study, we investigated the effects of different polarized macrophages on EMT of HMrSV5 PMCs. Methods: Monocytes were polarized to M1/M2 macrophages before being added to HMrSV5 in direct or indirect contact. Morphological changes of HMrSV5 were observed and toll-like receptors 4 (TLR4) on macrophage surfaces was detected using flow cytometry. EMT markers and intracellular signals of HMrSV5 cells were assessed using real time-PCR and WB. Results: The typical epithelial cell morphology of HMrSV5 disappeared after co-culture with M1 macrophages and was accompanied by decreased E-cadherin and increased $\alpha$-SMA, suggesting HMrSV5 undergo EMT. These effects depended on direct contact between the two cells, as indirect contact or co-culture with M2 macrophages had no effect. Intriguingly, we found TLR4 surface receptors were activated on sorted $\mathrm{M} 1$ cells in co-culture, and related signal adaptors, such as TRIF, were obviously upregulated. Conclusion: Direct contact with M1 macrophages induces EMT of PMCs, during which TRIF-dependent TLR4 signaling pathway was activated.
\end{abstract}

(C) 2016 The Author(s)

Published by S. Karger AG, Basel

\section{Introduction}

Continuous ambulatory peritoneal dialysis (CAPD) is a renal replacement therapy for patients with end-stage renal disease (ESRD). Compared with hemodialysis (HD), CAPD has shown several advantages in the continuous removal of waste products and retention of residual renal function that result in higher quality of life. Unfortunately, recurrent peritonitis and progressive peritoneal fibrosis (PF) ultimately lead to ultrafiltration failure. Patients with PD-related complications have to quit dialysis after several years of therapy [1]. 


\section{Cellular Physiology Cell Physiol Biochem 2016;40:1538-1548 \begin{tabular}{l|l|l} 
and BOI: 10.1159/000453204 & $\begin{array}{l}\text { (c) 2016 The Author(s). Published by S. Karger AG, Basel } \\
\text { www.karger.com/cpb }\end{array}$ \\
\hline Published online: December 21, 2016
\end{tabular} \\ Shi et al.: TLR4 in M1-Induced EMT}

Chronic inflammation plays a key role in peritoneal fibrosis. Macrophages are tissue sentinels that maintain tissue integrity by eliminating or repairing damaged cells and matrices. In addition, macrophages exhibit a range of phenotypes, a phenomenon that has been described as macrophage polarization or heterogeneity [2, 3]. The 'classically' activated M1 macrophage expresses pro-inflammatory cytokines such as IL-1, TNF-a, and IL-6. In contrast, the alternatively activated M2 macrophage produces anti-inflammatory molecules [4-6]. Distinct macrophage phenotypes contribute to kidney injury and repair [7, 8]. However, the function of macrophages in the inflammatory microenvironment during PD is still unknown.

Epithelial-mesenchymal transition (EMT), a process in which epithelial cells lose their epithelial phenotype and acquire fibroblast-like characteristics, also plays a crucial role in PF [9]. In this process, epithelial cell markers such as E-cadherin are diminished, while mesenchymal cell markers such as $\alpha$-SMA are upregulated. Our previous study demonstrated that monocyte infiltration could directly induce proximal tubular EMT [10], but there is no direct evidence that monocytes/macrophages regulate EMT in PMCs.

Toll-like receptors (TLRs) are important in immune response and are primarily expressed on macrophages. Of these receptors, TLR4 has been the most-studied in macrophages polarization and peritonitis $[11,12]$. It is suggested that TLR4 signaling augments TGF- $\beta$ synthesis and is implicated in fibrogenesis [13]. Some studies have also demonstrated that TLR4 contributes to the resolution of postinflammatory fibrogenesis [14]. To observe the effect and mechanism of different polarized macrophages on EMT of PMCs, we established a cell co-culture model in the present study. Our results suggest that M1 macrophages induce EMT of PMCs through the TLR4 signaling pathway.

\section{Materials and Methods}

\section{Cell lines and culture conditions}

Human peritoneal mesothelial cells (HMrSV5) were cultured in DMEM (Hyclone, Logan, UT, USA) supplemented with $10 \%$ heat-inactivated fetal calf serum (FCS; Invitrogen, Carlsbad, CA, USA) and $100 \mathrm{U} / \mathrm{ml}$ penicillin/streptomycin (Invitrogen). THP-1 cells, originally derived from human monocytic leukemia, were grown in suspension culture in RPMI 1640 medium (Hyclone) supplemented with 10\% FCS and penicillin/ streptomycin. Cells were incubated at $37^{\circ} \mathrm{C}$ in $5 \% \mathrm{CO}_{2}$, and the culture medium was replaced every 2 days. All experiments were carried out $24-48 \mathrm{~h}$ after cells were seeded in culture plates. Cells were permitted to attach for $24 \mathrm{~h}$ and to grow to $75 \%$ confluence.

Differentiation and polarization of monocyte-derived macrophages and co-culture with HMrSV5 cells

$5 \times 10^{5}$ /well monocytes were maintained in six-well plates and supplemented with $10 \%$ FCS and 200 ng/ml PMA (Sigma-Aldrich, St Louis, MO, USA) for $72 \mathrm{~h}$ to generate macrophages (labeled as M0). Cells were then washed, and the medium was replaced. Macrophages were polarized to M1 after treatment with 20 ng/ml IFN- $\gamma$ (Peprotech, Rocky Hill, NJ, USA) and 100 ng/ml LPS (Sigma-Aldrich) for 24 h. M2 macrophages were generated by stimulating M0 macrophages with $20 \mathrm{ng} / \mathrm{ml}$ recombinant human IL-4 (Peprotech) for 24 h. After 4 days, M0, M1 or M2 macrophages were completely differentiated. For co-culture of HMrSV5 cells and relevant macrophages, adherent HMrSV5 cells were washed once with serum-free DMEM and detached. $1 \times 10^{6} /$ well cells were added to the macrophage monolayer. To further examine the influence of direct or indirect contact with macrophages, we constructed an indirect contact co-culture model with Transwell plates (6-well, 8- $\mu$ m pore size; Millipore, Bedford, MA, USA). HMrSV5 cells were seeded on the upper chamber at a density of $5 \times 10^{5}$ cells $/ \mathrm{ml}$ and incubated with macrophages in the lower chamber in serum-free DMEM for $24 \mathrm{~h}$.

ELISA

Macrophage-secreted cytokines in the culture medium were detected with ELISA kits for human IL-6 and IL-10 (R\&D Systems, Minneapolis, MN, USA) according to the manufacturer's instructions. 


\section{Cellular Physiology Cell Physiol Biochem 2016;40:1538-1548 \begin{tabular}{l|l|l} 
and Biochemistry $\begin{array}{l}\text { DOI: 10.1159/000453204 } \\
\text { Published online: December 21, } 2016\end{array}$ & $\begin{array}{l}\text { (c) } 2016 \text { The Author(s). Published by S. Karger AG, Basel } \\
\text { www.karger.com/cpb }\end{array}$
\end{tabular} \\ Shi et al.: TLR4 in M1-Induced EMT}

Real-time PCR

Total RNA in treated cells was extracted by TRIzol reagent (Life Technologies, Gaithersburg, MD, USA) according to the manufacturer's instructions. cDNA was then synthesized by using a reverse transcription (RT) system kit (Takara, Japan). Real-time PCR was performed on ABI PRISM 7500 real-time PCR System (Applied Biosystems, Carlsbad, CA, USA). The PCR primers were designed as follows: iNOs: 5'-AGC CTG TGA GAC GTT TGA TGT-3' 5'-TGT AGA TTC TGC CGA GAT TTGA-3'; TNF- $\alpha$ : 5'-TTG AGG GTT TGC TAC AAC ATGGG-3' 5'-GCT GCA CTT TGG AGT GATCG-3'; CXCL10: 5'-CAA ATC TGC TTT TTA AAG AAT GCTC-3' 5'-AAG AAT TTG GGC CCC TTG-3'; Arg-1: 5'-GGC TGG TCT GCT TGA GAAAC-3' 5'-ATT GCC AAA CTG TGG TCTCC-3'; CD163: 5'-CGG CTG CCT CCA CCT CTA AGT-3' 5'-ATG AAG ATG CTG GCG TGACA-3'; a-SMA: 5'-GAC AAT GGC TCT GGG CTC TGTAA-3' 5'-ATG CCA TGT TCT ATC GGG TAC TTCA-3'; E-cadherin: 5'-GGA TTG CAA ATT CCT GCC ATTC3' 5'-AAC GTT GTC CCG GGT GTC AAG-3'; TRIF:5'-CAG GAG CCT GAG GAG ATGAG-3' 5'-CTG GGT AGT TGG TGC TGGTT-3'; IRF3: 5'-CCC TCT GAG AAC CCA CTGAA-3' 5'-TGC CTC ACG TAG CTC ATCAC-3'; IKK: 5'-CTG TTC TGT GGC TGC CTGTA -3' 5'-GAG AAG CAG GTC CTT TCGTG-3'; GAPDH: 5'-GCA CCG TCA AGG CTG AGAAC-3' 5'-ATG GTG GTG AAG ACG CCAGT-3'. All mRNA quantification data were normalized to human GAPDH as an endogenous control for mRNA detection. The data were processed using the $2^{-\Delta \Delta C t}$ method.

\section{Western blot analysis}

Total proteins were extracted from treated cells using RIPA buffer (Thermo Fisher Scientific, USA) according to the manufacturer's instructions and measured using the BCA Protein Assay Kit (Thermo Fisher Scientific). Equal amounts of proteins ( $25 \mu \mathrm{g} / \mathrm{lane}$ ) were separated using SDS-PAGE and electro transferred onto PVDF membranes. The membranes were blocked with 5\% BSA in TBS containing 0.5\% Tween 20 (TBST) at room temperature for $2 \mathrm{~h}$. Then, membranes were incubated with primary antibodies at $4^{\circ} \mathrm{C}$ overnight, followed by incubation with HRP-conjugated goat anti-mouse or anti-rabbit IgG secondary antibodies (Santa Cruz, CA, USA) for $1 \mathrm{~h}$. Finally, signals were detected using ECL (Millipore). The primary antibodies included E-cadherin (CST, Beverly, MA, USA), $\alpha$-SMA (Abcam, Cambridge, UK), TRIF (CST), IRF3 (CST), IKK (CST) and GAPDH (CST). Expression levels were normalized to GAPDH.

Flow cytometry and fluorescence-activated cell sorting (FACS)

M1/M2 macrophages were collected using centrifugation and resuspended in $200 \mu \mathrm{l}$ of PBS. Then, cells were incubated on ice for $30 \mathrm{~min}$ in the dark with PE-conjugated anti-human TLR4 (eBioscience, San Diego, USA) in $200 \mu \mathrm{l}$ of binding buffer (PBS supplemented with $5 \mathrm{mM} \mathrm{MgCl} 2,4.5 \mathrm{~g} / \mathrm{l}$ glucose, and $1 \mathrm{mg} / \mathrm{ml}$ BSA). Cells were washed with PBS, suspended in $200 \mu \mathrm{l}$ of binding buffer and analyzed using flow cytometry (BD FACS Aria III; BD Bioscience, San Jose, CA, USA). In the co-culture model, CD14+ (eBioscience), TLR4+ and CD14+/TLR4+ double-labeled cells were sorted. Wild M1/M2 cells were also sorted to eliminate the effect of sorting procedure on membrane receptors expression. Then, TLR4+ cells were analyzed again among the separated cells.

\section{Statistical analysis}

All data from at least three independent experiments were expressed as the means \pm standard error of the mean (SEM) and analyzed using one-way ANOVA with SPSS 19.0 statistical software. Values of $\mathrm{P}<0.05$ were considered statistically significant.

\section{Results}

\section{Successful differentiation of M1/M2 macrophages}

As shown in Fig. 1A, THP-1 cells grew in suspension. Upon stimulation with $200 \mathrm{ng} / \mathrm{ml}$ PMA for $72 \mathrm{~h}, \mathrm{M} 0$ macrophages were generated that adhered to the wall and had outstretched

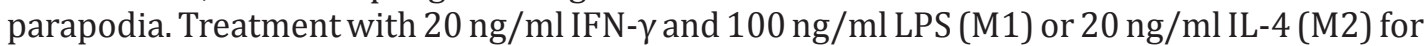
another $24 \mathrm{~h}$ resulted in a vigorous growth of macrophages, larger cell bodies and extended parapodia. Compared with native macrophages, the expression of IL-6 was increased in M1 macrophages, and the expression of IL-10 was higher in M2 macrophages (Fig. 1B; ${ }^{*} \mathrm{P}<0.05$ vs. control). Furthermore, M1 macrophages showed considerably higher expression of iNOs, TNF- $\alpha$ and CXCL10 mRNA than native macrophages (M0) (Fig. 1 C; ${ }^{*}$ P<0.05 vs. control). The 
A
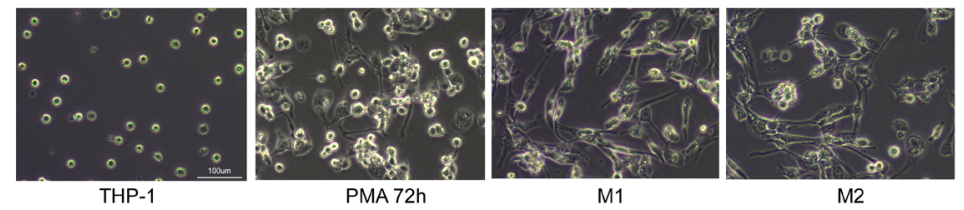

B
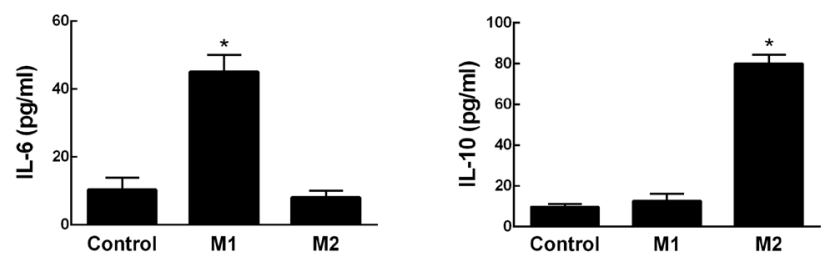

C

D
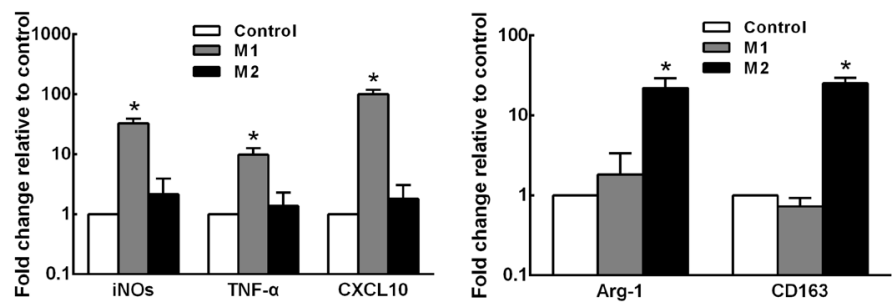

Fig. 1. Monocytes differentiated into M0/M1/M2 macrophages. (A) Phase contrast microscope images of the phenotypic change of cells. After stimulation with $200 \mathrm{ng} / \mathrm{ml}$ PMA for $72 \mathrm{~h}$, suspended THP-1 monocytes differentiated into M0 macrophages that adhered to the wall and had outstretched parapodia. After treating with $20 \mathrm{ng} / \mathrm{ml} \mathrm{IFN-} \gamma$ and $100 \mathrm{ng} / \mathrm{ml} \mathrm{LPS} \mathrm{(M1)} \mathrm{or} 20 \mathrm{ng} / \mathrm{ml} \mathrm{IL-4} \mathrm{(M2)} \mathrm{for} \mathrm{another} 24 \mathrm{~h}$, macrophages presented with larger cell bodies and longer parapodia $(10 \times 20)$. (B) ELISA of the secreted cytokines showed that the expression of IL- 6 was significantly upregulated in M1 macrophages and the expression of IL-10 was significantly increased in M2 macrophages ( ${ }^{\mathrm{P}}<0.05$ vs. control). (C) Real-time PCR showed that mRNA levels of iNOs, TNF- $\alpha$, and CXCL10 were significantly upregulated in M1 cells, while mRNA levels of Arg-1 and CD163 were significantly increased in M2 cells ( ${ }^{*} \mathrm{P}<0.05$ vs. control).

mRNA expression of Arg-1 and CD163 in M2 macrophages was significantly higher than in native macrophages (Fig. $1 \mathrm{D} ;{ }^{*} \mathrm{P}<0.05$ vs. control). These results suggest successful induction of different polarized macrophages. M1/M2 macrophages were co-cultured with PMCs.

Direct contact with M1 macrophages induced fibroblast morphology of PMCs

PMCs were grown in monoculture and then grown in direct contact with M1/ M2 macrophages. The morphological alterations were observed under phase-contrast microscopy. PMCs co-cultured with M1 macrophages developed a series of phenotypic changes including elongation, branching, and loss of cobblestone-like features. In contrast, PMCs co-cultured with M2 macrophages did not undergo significant morphological change (Fig. 2A).

Direct contact with M1 macrophages upregulated the expression of mesenchymal markers but downregulated epithelial markers in PMCs

We next examined whether direct co-culture with M1 macrophages altered EMT markers in PMCs. Using real-time PCR analysis, we found that the mRNA expression of the epithelial marker E-cadherin was decreased in PMCs directly co-cultured with M1 macrophages 
A

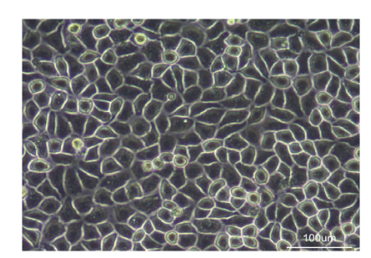

Control(HMrSV5)

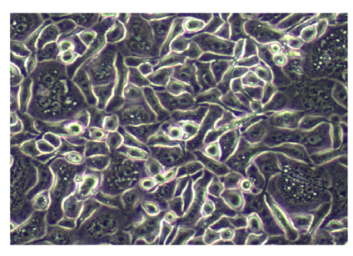

M1+HMrSV5

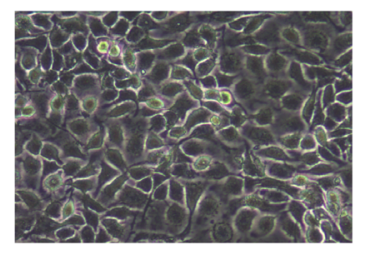

M2+HMrSV5

B

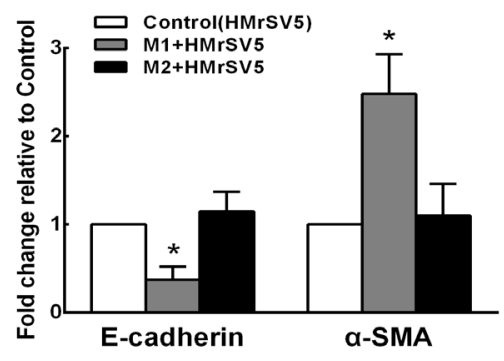

C
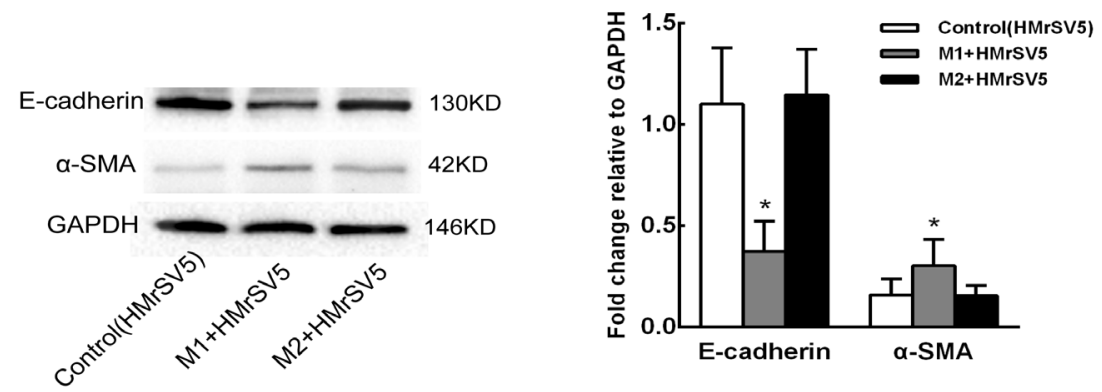

Fig. 2. The effects of direct co-culture of PMCs with macrophages on EMT. (A) Phase contrast microscope images of the phenotypic change of HMrSV 5 cells in direct co-culture with macrophages. The normal phenotypes of HMrSV5 cells disappeared after co-culture with M1 cells for $24 \mathrm{~h}$ : HMrSV5 cells developed a series of phenotypic changes including elongation, branching, and loss of cobblestone appearance. No dramatic difference was observed after co-culture with M2 cells $(10 \times 20)$. (B) Real-time PCR showed that when HMrSV5 cells were directly co-cultured with M1 cells, E-cadherin mRNA expression decreased, while $\alpha$-SMA mRNA expression increased $(* \mathrm{P}<0.05$ vs. control). In contrast, direct co-culture with M2 macrophages did not affect the expression of E-cadherin or $\alpha$-SMA. (C) Western blotting produced similar results to PCR ( ${ }^{*} \mathrm{P}$ $<0.05$ vs. control).

compared to PMCs in monoculture, while the mRNA expression of the mesenchymal marker $\alpha$-SMA was significantly higher (Fig. $2 \mathrm{~B}$; ${ }^{*} \mathrm{P}<0.05$ vs. control). Furthermore, Western blotting showed that E-cadherin protein expression was similarly decreased, while $\alpha$-SMA protein expression was significantly higher, in PMCs directly co-cultured with M1 macrophages than in native PMCs (Fig. $2 \mathrm{C} ;{ }^{*} \mathrm{P}<0.05$ vs. control). Taken together, these data strongly suggest that PMCs undergo EMT upon direct co-culture with M1 macrophages. However, both realtime PCR and Western blotting results indicated that direct co-culture with M2 macrophages did not affect the expression of EMT markers in PMCs (Fig. $2 \mathrm{~B}$ and $\mathrm{C}$; ${ }^{*} \mathrm{P}<0.05$ vs. control).

PMCs failed to undergo EMT upon indirect co-culture with M1/M2 macrophages

We established an indirect co-culture model to explore the possibility that the EMTpromoting effect of M1 macrophages on PMCs in the direct co-culture model may be due to 
Fig. 3. PMCs failed to undergo EMT upon indirect co-culture with M1/M2 macrophages. (A) Phase contrast microscope images of HMrSV5 cells in upper Transwell chambers. No alteration of epithelial cell phenotype presented when HMrSV5 cells were indirectly co-cultured with M1/M2 cells. (B) According to real-time PCR, there was no significant difference in the mRNA expression of E-cadherin or $\alpha$-SMA between wild type HMrSV5 and those indirectly co-cultured with M1 or M2. (C) Relative protein expression measured using Western blotting was similar to mRNA.

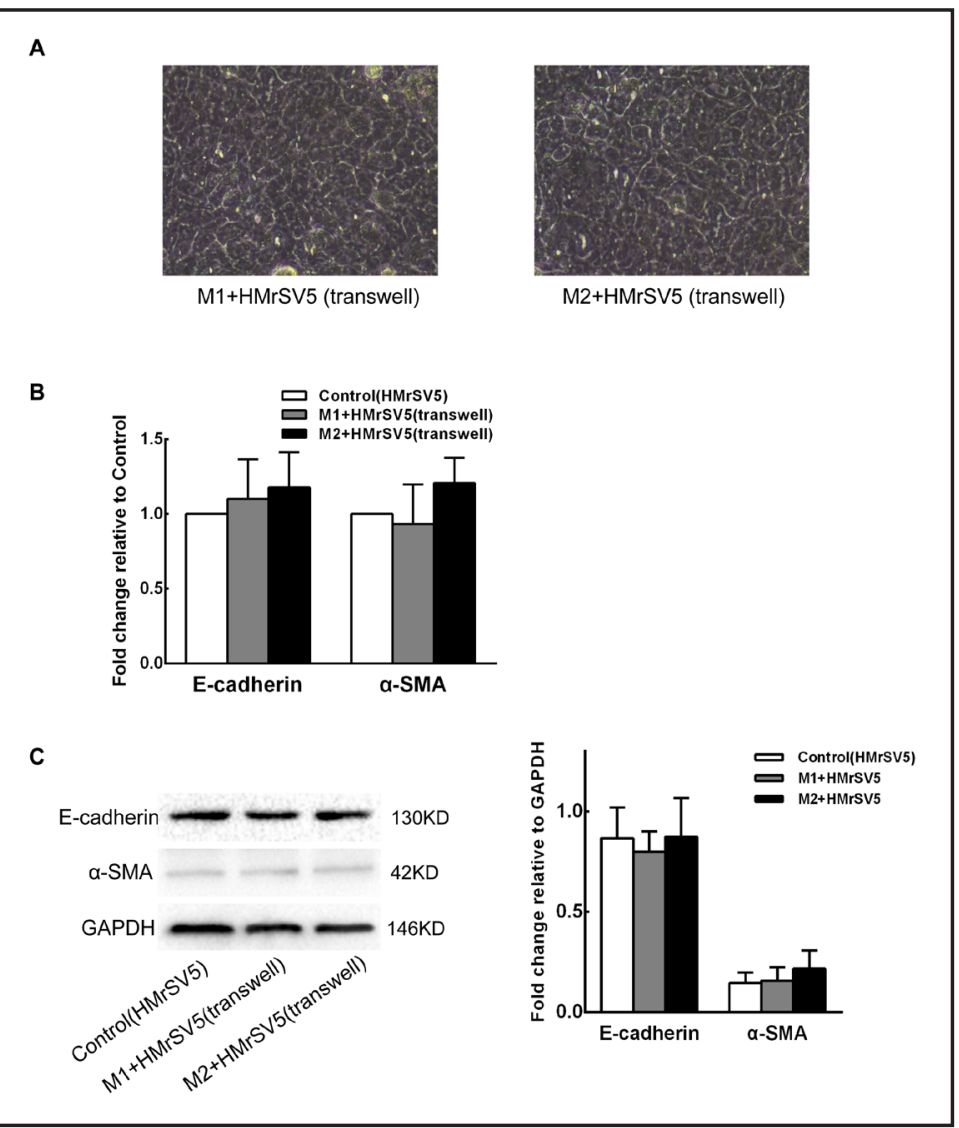

soluble factors secreted by the macrophages. PMCs were seeded into the upper Transwell chambers and co-cultured with either M1 or M2 macrophages in the lower chamber. After incubation for 24 hours, morphological alterations were observed, and relative EMT markers were detected in PMCs. Interestingly, we found that neither M1 nor M2 macrophages indirectly co-cultured with PMCs induced phenotype changes in PMCs (Fig. 3A). Real-time PCR and Western blot analysis also revealed no significant difference in the expression of E-cadherin or $\alpha$-SMA at both the mRNA (Fig. 3B) and protein levels (Fig. 3C) between native PMCs and PMCs in the indirect co-culture model (M1 and M2). Altogether, these results suggest that cytokines or soluble factors released from macrophages are not likely responsible for EMT of PMCs.

Membrane expression of TLR4 on M1 macrophages was activated in EMT of PMCS

Cell-cell contact between M1 macrophages and PMCs induced the EMT process. Next, we used FACS to separate macrophages from the systems with or without co-culture by sorting TLR4+ or CD14+ cells (Fig. 4A). Membrane TLR4 expression was detected in M1/ M2 macrophages from different sources (Fig. 4B). The results showed that compared with sorted M1 cells without co-culture, M1 macrophages directly co-cultured with PMCs expressed a higher level of TLR4 on their cell surface $(92.2 \pm 6.5 \%$ vs $56.4 \pm 7.8 \%$, $* * \mathrm{P}<$ 0.01 ), while TLR4 expression on the surface of M1 macrophages co-cultured with PMCs in transwell chambers increased slightly but not significantly (Fig. 4C; $68.0 \pm 10.8 \%$ vs 56.4 $\pm 7.8 \%, \mathrm{p}>0.05$ ). Furthermore, the percentage of TLR4+ cells did not significantly change between native and co-cultured M2 macrophages in both the direct (56.0 $\pm 9.8 \%$ vs $46.3 \pm$ $10.7 \%, \mathrm{P}>0.05)$ and indirect contact model $(54.3 \pm 12.8 \%$ vs $46.3 \pm 10.7 \%, \mathrm{P}>0.05)$ (Fig. 4C). Thus, TLR4 was externally expressed on macrophages activated by direct co-culture of PMCs and M1 macrophages. 
Fig. 4. Membrane TLR4 on M1 $\mathrm{m}$ acrophages was activated in the EMT of PMCs. (A) TLR4+ or CD14+ cells were sorted using FACS to separate macrophages from the systems with or without co-culture. (B) Flow cytometry was used to detect membrane TLR4 expression in sorted M1/M2 and cells in transwells. (C) Statistical analysis showed M1 macrophages directly co-cultured with PMCs express a higher level of TLR4 on the cell surface than controls. Data are presented as the mean \pm s.d. and are representative of three independent experiments (**P $<0.01$ compared with indicated groups).

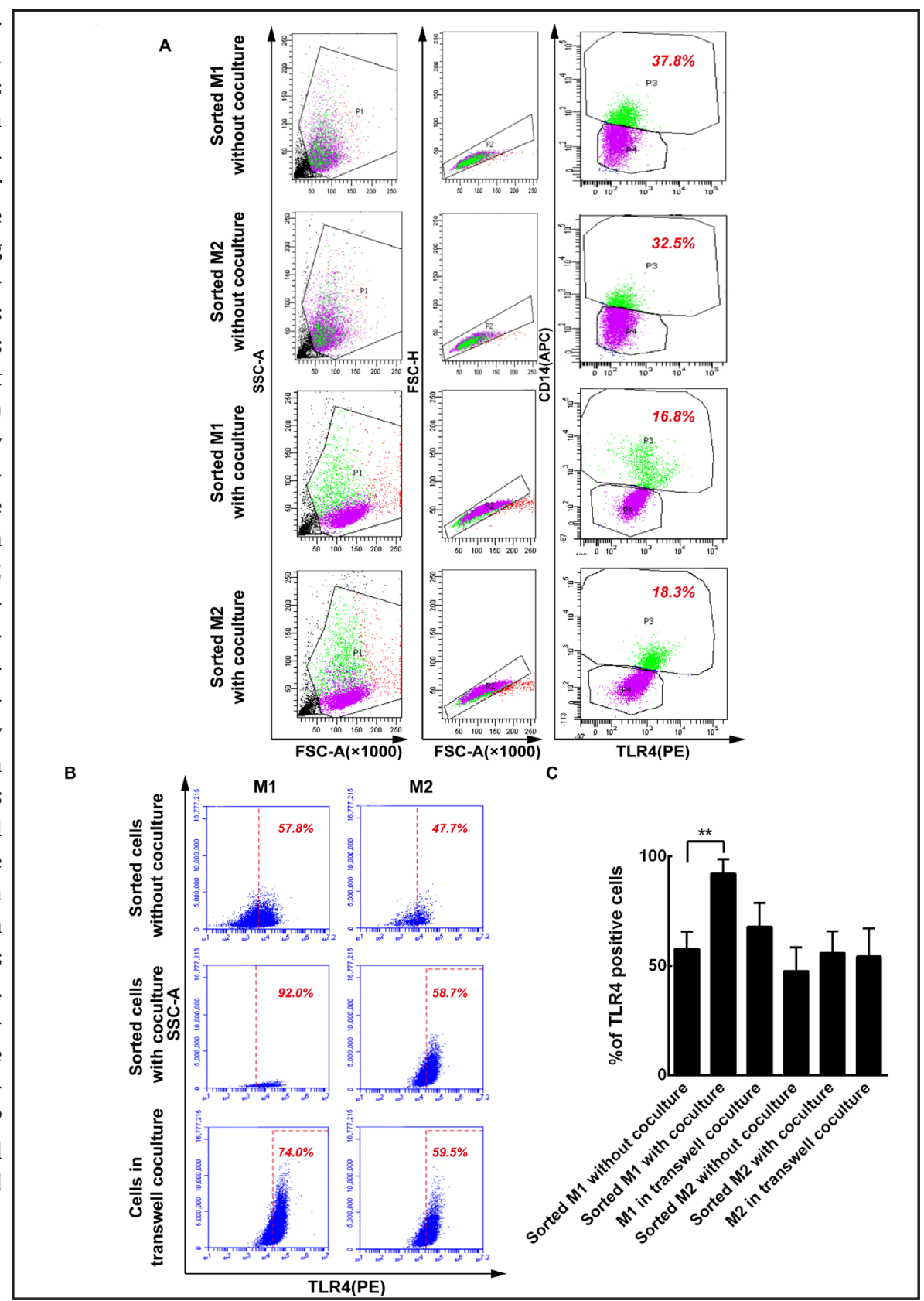

Activation of TRIF-dependent TLR4 signaling pathway in M1-induced EMT of PMCs

Finally, we examined the activation of the TLR4 signaling pathway in M1 macrophages during EMT of PMCs. Real-time PCR analysis indicated that the mRNA levels of TRIF, IRF3 and IKK in sorted M1 macrophages were significantly increased compared to wild types (Fig. $5 \mathrm{~A} ;{ }^{*} \mathrm{P}<0.05$ vs. control). Western blotting also showed increased relative protein expression in M1 macrophages that directly contacted PMCs (Fig. 5B; ${ }^{*} \mathrm{P}<0.05$ vs. control).

\section{Discussion}

Peritoneal fibrosis is the most common change observed in the peritoneal tissues of patients who undergo long-term PD therapy. The morphological change of the peritoneal 
A

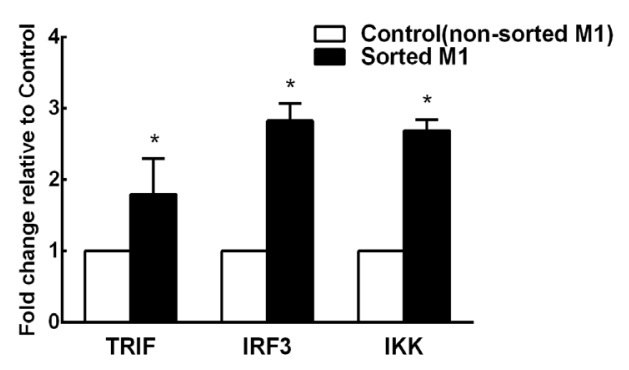

B
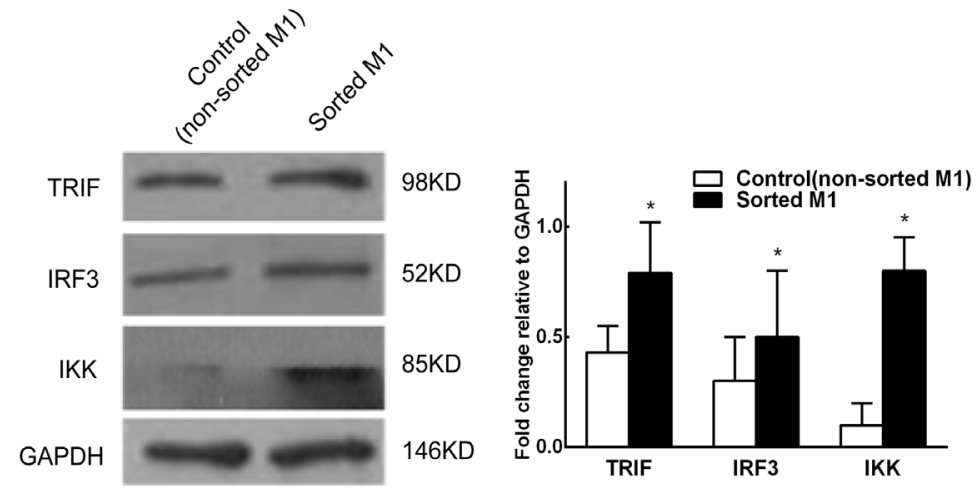

Fig. 5. TRIF-dependent TLR4 signaling pathway was activated in M1-induced EMT of PMCs. (A) Real-time PCR analysis indicated that mRNA levels of TRIF, IRF3 and IKK in sorted M1 macrophages were significantly increased compared to wild types ( ${ }^{*} \mathrm{P}<0.05$ vs. control). (B) Western blotting indicated similar results to PCR $\left({ }^{*} \mathrm{P}<0.05\right.$ vs. control).

membrane is characterized by a detachment of the mesothelial layer, angiogenesis, a progressive submesothelial thickening with ECM deposition and an increased presence of myofibroblasts. EMT of mesothelial cells contributes to the initiation of this process and plays a reversible role. Yanez-Mo and colleagues first demonstrated that PMCs in patients with PD underwent a transition from an epithelial to a mesenchymal phenotype [15], and the potential role of EMT in peritoneal fibrosis has been established over the last 10 years $[16,17]$. Targeting EMT of PMCs is thought to be a potential therapeutic strategy for delaying $\mathrm{PF}$ and improving efficiency of PD.

In the context of fibrosis, it is believed that EMT begins as a pro-fibrogenic event that normally generates fibroblasts and other related cells to reconstruct tissues following inflammatory injury, but ceases once inflammation is attenuated. However, EMT can pathologically continue to respond to ongoing inflammation, eventually leading to tissue structure destruction (fibrosis) [18]. Both noninfectious and infectious inflammation can cause PF and ultrafiltration failure. Data shows that despite clinical remission, patients with PD still suffer from prolonged systemic chronic inflammation [19]. Injured PMCs and resident macrophages recruit inflammatory cells such as monocytes and lymphocytes, which secrete high levels of pro-inflammatory cytokines that could, in turn, continuously trigger an inflammatory response in the peritoneum [20]. Patients undergoing CAPD have increased levels of pro-inflammatory cytokines, including IL-1 $\beta$, IL-6, TNF- $\alpha$, and TGF- $\beta$, in both PD effluent and plasma [21].

There is a strong correlation between macrophages and EMT of PMCs, however, previous studies have focused on the pro-EMT effect of different cytokines or molecules, not macrophages. Furthermore, the polarized alteration of recruited monocytes during EMT of PMCs is also unclear. Here, we showed for the first time that macrophages are involved in EMT of PMCs. We used classical methods to differentiate THP-1 human leukemia monocytes 
into polarized macrophages (M0 (native state), M1, M2). M1 cells expressed higher levels of IL-6, iNOs and TNF- $\alpha$, while M2 cells expressed higher levels of IL-10, Arg-1 and CD163, similar to other reports $[22,23]$. We demonstrated that direct co-culture of M1 macrophages and HMrSV5 cells induced a phenotypic change in HMrSV5 characterized by cell elongation, branching, and loss of cobblestone-like appearance. In addition, co-culture induced the downregulation of E-cadherin and upregulation of $\alpha$-SMA in HMrSV5 cells, suggesting that M1 macrophages promote the phenotypic change of PMCs via EMT. M2 macrophages, in contrast, had no effect on EMT of PMCs.

To clarify whether the effect was induced by cytokines and other soluble molecules secreted by M1 macrophages or by direct contact between M1 cells and HMrSV5 cells, an indirect co-culture model was built using Transwell chambers. Intriguingly, our results suggested that the conditioned medium of M1/M2 macrophages had no significant effects on HMrSV 5 cells. Instead of soluble factors secreted by macrophages, it is direct cell-cell contact between M1 cells and HMrSV5 cells that plays a key role in EMT.

What is the mechanism of M1 macrophage-mediated EMT in PMCs? TLR4 is a recognized inflammatory regulator and seems to participate in fibrogenesis [13]. To the best of our knowledge, no studies have addressed whether surface expression of TLR4 on macrophages promotes EMT. CD14, the co-receptor of TLR4, is also a macrophage-specific cell surface marker [24]. In the presence of inflammation, the TLR4-MD2-CD14 complex was recruited to the macrophages surface, and downstream pathways were activated [25]. In our study, wild macrophages and cells in the co-culture system were separated using FACS. The surface expression of TLR4 in M1 macrophages increased markedly when directly co-cultured with HMrSV5 cells but not in the indirect co-culture system or co-culture with M2 cells. All the above indicate that the surface receptor of M1 macrophages, TLR4, is activated during EMT of PMCs. To confirm TLR4 signaling activity during EMT of PMCs, the adaptors that facilitate TLR4 signal transduction were investigated. TRIF, one of the distinct adaptor proteins, first engages to TLR4, then transcription factor IRF3 is activated and induces the expression of IKK [26]. In the current study, direct co-culture of M1 macrophages and HMrSV5 cells upregulated TRIF, IRF3 and IKK expression on M1 cells, suggesting activation of TRIFdependent TLR4 signaling in EMT of PMCs.

Though TLR4 signaling is activated in macrophages, in this study, unfortunately, the receptors and downstream signaling change in PMCs during M1-dependent EMT is not observed, as well as the different response between M1 and M2 cells. It has been reported that several signaling pathways or growth factors (TGF $\beta 1 /$ Smads, Wnt/ $\beta$-catenin, and Notch) participate in EMT [27], among which TGF- $\beta 1$ is the well-recognized induced factor of EMT and subsequent fibrosis [28]. Our previous studies also revealed that TGF- $\beta 1$ was highly upregulated in EMT of peritoneum in vivo and HMrSV5 cells underwent EMT after treatment by TGF- $\beta 1[29,30]$. In line with this view, several studies support that TLR4 activation regulates the TGF- $\beta 1$ pathway $[31,32]$. Since M1-dependent EMT of PMCs is irrelevant to the secreted cytokines, the interactions between TLR4-activated M1 cells and PMCs require elucidation.

In summary, we have identified a novel relationship between macrophages and PMCs during EMT. Specifically, it is direct cell-cell contact of M1 macrophages promotes EMT in PMCs. We also demonstrated that TRIF-dependent TLR4 signaling in M1 cells is obviously activated in this process. Further studies that aim to illustrate underlying cell-cell crosstalk between macrophages and PMCs should be performed.

\section{Acknowledgments}

This work was supported by grants from the National Natural Science Foundation of China (No.81300631, No.81200556), the Natural Science Foundation of Jiangsu Province (BK20131036) and the Jiangsu Province Traditional Chinese Medicine Hospital peak talent fund (y2014rc15). 


\section{Cellular Physiology Cell Physiol Biochem 2016;40:1538-1548

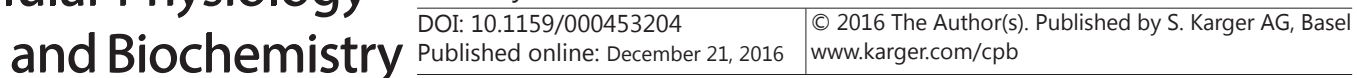 Shi et al.: TLR4 in M1-Induced EMT}

\section{Disclosure Statement}

All authors have no conflicts of interest to declare.

\section{References}

1 Jain AK, Blake P, Cordy P, Garg AX: Global trends in rates of peritoneal dialysis. J Am Soc Nephrol 2012;23:533-544.

2 Van Ginderachter JA, Movahedi K, Hassanzadeh Ghassabeh G, Meerschaut S, Beschin A, Raes G, De Baetselier P: Classical and alternative activation of mononuclear phagocytes: picking the best of both worlds for tumor promotion. Immunobiology 2006;211:487-501.

3 Martinez FO, Helming L, Gordon S: Alternative activation of macrophages: an immunologic functional perspective. Annu Rev Immunol 2009;27:451-483.

4 Wang Y, Wang YP, Zheng G, Lee VW, Ouyang L, Chang DH, Mahajan D, Coombs J, Wang YM, Alexander SI, Harris DC: Ex vivo programmed macrophages ameliorate experimental chronic inflammatory renal disease. Kidney Int 2007;72:290-299.

5 Mantovani A, Sica A, Sozzani S, Allavena P, Vecchi A, Locati M: The chemokine system in diverse forms of macrophage activation and polarization. Trends Immunol 2004;25:677-686.

6 Wynn TA: Fibrotic disease and the T(H)1/T(H)2 paradigm. Nat Rev Immunol 2004;4:583-594.

7 Ricardo SD, van Goor H, Eddy AA: Macrophage diversity in renal injury and repair. J Clin Invest 2008;118:3522-3530.

8 Pan B, Liu G, Jiang Z, Zheng D: Regulation of renal fibrosis by macrophage polarization. Cell Physiol Biochem 2015;35:1062-1069.

9 Kalluri R, Neilson EG: Epithelial-mesenchymal transition and its implications for fibrosis. J Clin Invest 2003;112:1776-1784.

10 Li Q Liu BC, Lv LL, Ma KL, Zhang XL, Phillips AO: Monocytes induce proximal tubular epithelialmesenchymal transition through NF-kappa B dependent upregulation of ICAM-1. J Cell Biochem 2011;112:1585-1592.

11 Kato S, Yuzawa Y, Tsuboi N, Maruyama S, Morita Y, Matsuguchi T, Matsuo S: Endotoxin-induced chemokine expression in murine peritoneal mesothelial cells: the role of toll-like receptor 4. J Am Soc Nephrol 2004;15:1289-1299.

12 Zhou Y, Zhang T, Wang X, Wei X, Chen Y, Guo L, Zhang J, Wang C: Curcumin Modulates Macrophage Polarization Through the Inhibition of the Toll-Like Receptor 4 Expression and its Signaling Pathways. Cell Physiol Biochem 2015;36:631-641.

13 Bhattacharyya S, Kelley K, Melichian DS, Tamaki Z, Fang F, Su Y, Feng G, Pope RM, Budinger GR, Mutlu GM, Lafyatis R, Radstake T, Feghali-Bostwick C, Varga J: Toll-like receptor 4 signaling augments transforming growth factor-beta responses: a novel mechanism for maintaining and amplifying fibrosis in scleroderma. Am J Pathol 2013;182:192-205.

14 Yang HZ, Wang JP, Mi S, Liu HZ, Cui B, Yan HM, Yan J, Li Z, Liu H, Hua F, Lu W, Hu ZW: TLR4 activity is required in the resolution of pulmonary inflammation and fibrosis after acute and chronic lung injury. Am J Pathol 2012;180:275-292.

15 Yanez-Mo M, Lara-Pezzi E, Selgas R, Ramirez-Huesca M, Dominguez-Jimenez C, Jimenez-Heffernan JA, Aguilera A, Sanchez-Tomero JA, Bajo MA, Alvarez V, Castro MA, del Peso G, Cirujeda A, Gamallo C, SanchezMadrid F, Lopez-Cabrera M: Peritoneal dialysis and epithelial-to-mesenchymal transition of mesothelial cells. N Engl J Med 2003;348:403-413.

16 Aroeira LS, Aguilera A, Sanchez-Tomero JA, Bajo MA, del Peso G, Jimenez-Heffernan JA, Selgas R, LopezCabrera M: Epithelial to mesenchymal transition and peritoneal membrane failure in peritoneal dialysis patients: pathologic significance and potential therapeutic interventions. J Am Soc Nephrol 2007;18:20042013.

17 Xiang S, Li M, Xie X, Xie Z, Zhou Q Tian Y, Lin W, Zhang X, Jiang H, Shou Z, Chen J: Rapamycin inhibits epithelial-to-mesenchymal transition of peritoneal mesothelium cells through regulation of Rho GTPases. FEBS J 2016;283:2309-2325. 


\section{Cellular Physiology Cell Physiol Biochem 2016;40:1538-1548

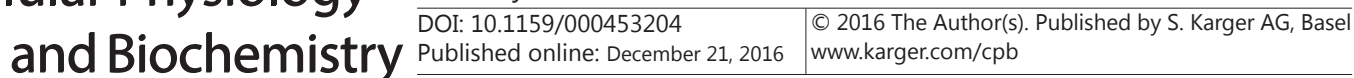 \\ Shi et al.: TLR4 in M1-Induced EMT}

18 Liu Y, Dong Z, Liu H, Zhu J, Liu F, Chen G: Transition of mesothelial cell to fibroblast in peritoneal dialysis: EMT, stem cell or bystander? Perit Dial Int 2015;35:14-25.

19 Lai KN, Leung JC: Inflammation in peritoneal dialysis. Nephron Clin Pract 2010;116:c11-18.

20 Baroni G, Schuinski A, de Moraes TP, Meyer F, Pecoits-Filho R: Inflammation and the peritoneal membrane: causes and impact on structure and function during peritoneal dialysis. Mediators Inflamm 2012;2012:912595.

21 Rosengren BI, Sagstad SJ, Karlsen TV, Wiig H: Isolation of interstitial fluid and demonstration of local proinflammatory cytokine production and increased absorptive gradient in chronic peritoneal dialysis. Am J Physiol Renal Physiol 2013;304:F198-206.

22 Jia X, Li X, Shen Y, Miao J, Liu H, Li G, Wang Z: MiR-16 regulates mouse peritoneal macrophage polarization and affects T-cell activation. J Cell Mol Med DOI:10.1111/jcmm.12882.

23 Lin S, Qiu M, Chen J: IL-4 Modulates Macrophage Polarization in Ankylosing Spondylitis. Cell Physiol Biochem 2015;35:2213-2222.

24 Wilensky A, Tzach-Nahman R, Potempa J, Shapira L, Nussbaum G: Porphyromonas gingivalis gingipains selectively reduce CD14 expression, leading to macrophage hyporesponsiveness to bacterial infection. J Innate Immun 2015;7:127-135.

25 Kawai T, Akira S: The role of pattern-recognition receptors in innate immunity: update on Toll-like receptors. Nat Immunol 2010;11:373-384.

26 O'Neill LA, Golenbock D, Bowie AG: The history of Toll-like receptors - redefining innate immunity. Nat Rev Immunol 2013;13:453-460.

27 Gonzalez DM, Medici D: Signaling mechanisms of the epithelial-mesenchymal transition. Sci Signal 2014;7:re8.

28 Strippoli R, Moreno-Vicente R, Battistelli C, Cicchini C, Noce V, Amicone L, Marchetti A, Del Pozo MA, Tripodi M: Molecular Mechanisms Underlying Peritoneal EMT and Fibrosis. Stem Cells Int 2016;2016:3543678.

29 Li Z, Zhang L, He W, Zhu C, Yang J, Sheng M: Astragalus membranaceus inhibits peritoneal fibrosis via monocyte chemoattractant protein (MCP)-1 and the transforming growth factor-beta1 (TGF-beta1) pathway in rats submitted to peritoneal dialysis. Int J Mol Sci 2014;15:12959-12971.

30 Zhang L, Li Z, He W, Xu L, Wang J, Shi J, Sheng M: Effects of Astragaloside IV Against the TGF-beta1-Induced Epithelial-to-Mesenchymal Transition in Peritoneal Mesothelial Cells by Promoting Smad 7 Expression. Cell Physiol Biochem 2015;37:43-54.

31 Stifano G, Affandi AJ, Mathes AL, Rice LM, Nakerakanti S, Nazari B, Lee J, Christmann RB, Lafyatis R: Chronic Toll-like receptor 4 stimulation in skin induces inflammation, macrophage activation, transforming growth factor beta signature gene expression, and fibrosis. Arthritis Res Ther 2014;16:R136.

32 Seki E, De Minicis S, Osterreicher CH, Kluwe J, Osawa Y, Brenner DA, Schwabe RF: TLR4 enhances TGF-beta signaling and hepatic fibrosis. Nat Med 2007;13:1324-1332. 